\title{
Multifunctional Magnetic and Upconverting Nanobeads as Dual Modal Imaging Tools
}

\author{
Maria Elena Materia, ${ }^{\ddagger \dagger}$ Manuel Pernia Leal, ${ }^{\ddagger \dagger, \S, \#}$ Marco Scotto, ${ }^{\dagger}$ Preethi Bala Balakrishnan, ${ }^{\dagger}$ \\ Sahitya Kumar Avugadda, ${ }^{\dagger}$ María L. García-Martín, ${ }^{\S}$ Bruce E. Cohen, ${ }^{\| \odot ~ E m o r y ~ M . ~ C h a n, ~}{ }^{\|}$ \\ and Teresa Pellegrino $*, \oplus$ \\ ${ }^{\dagger}$ Istituto Italiano di Tecnologia, Via Morego 30, 16163 Genova, Italy \\ ${ }^{\S}$ BIONAND, Andalusian Centre for Nanomedicine and Biotechnology (Junta de Andalucía-Universidad de Málaga), 29590 Málaga, \\ Spain \\ "The Molecular Foundry, Lawrence Berkeley National Laboratory, Berkeley, California 94720, United States
}

Supporting Information

ABSTRACT: We report the fabrication of aqueous multimodal imaging nanocomposites based on superparamagnetic nanoparticles (MNPs) and two different sizes of photoluminescent upconverting nanoparticles (UCNPs). The controlled and simultaneous incorporation of both types of nanoparticles (NPs) was obtained by controlling the solvent composition and the addition rate of the destabilizing solvent. The magnetic properties of the MNPs remained unaltered after their encapsulation into the polymeric beads as shown by the T2 relaxivity measurements. The UCNPs maintain photoluminescent properties even when embedded with the MNPs into the polymer bead. Moreover, the light emitted by the magnetic and upconverting nanobeads (MUCNBs) under NIR excitation $\left(\lambda_{\text {exc }}=980 \mathrm{~nm}\right)$ was clearly observed through different thicknesses of agarose gel or through a mouse skin layer. The comparison with magnetic and luminescent nanobeads based on red-emitting quantum dots (QDs) demonstrated that while the QD-based beads show significant autofluorescence background from the skin, the signal obtained by the MUCNBs allows a decrease in this background. In summary, these results indicate that MUCNBs are good magnetic and optical probes for in vivo multimodal imaging sensors.
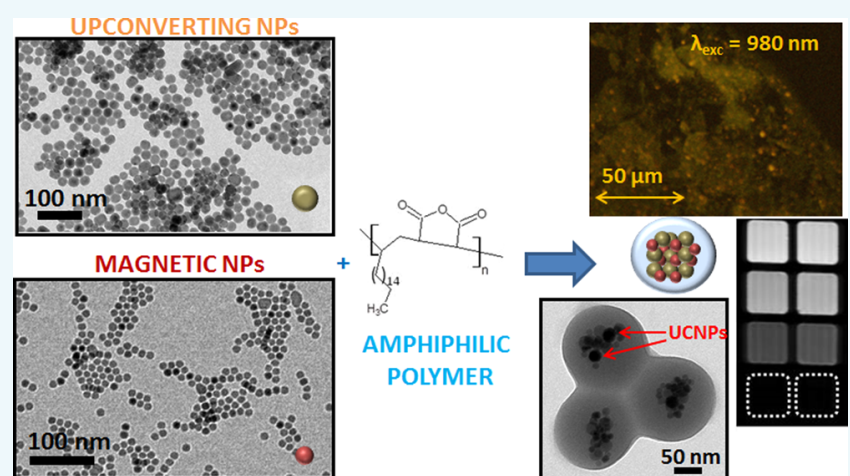

\section{INTRODUCTION}

Upconverting nanoparticles (UCNPs) have emerged as promising probes for biological imaging ${ }^{1-5}$ due to their exceptional capacity to emit ultraviolet and visible photons after being excited by lower-energy photons in the NIR range. High photochemical stability; deep tissue penetration; ${ }^{6}$ absence of autofluorescence; photobleaching; blinking ${ }^{7}$ and low toxicity in comparison with traditional fluorescent nanomaterials ${ }^{8}$ are other outstanding characteristics of UCNPs. The most efficient UCNPs are the $\beta$-phase $\mathrm{NaYF}_{4}$ doped with a $\mathrm{Yb}^{3+}$ sensitizer and $\mathrm{Fr}^{3+}$ emitter. In combination with other functional NPs such as gold $\mathrm{NPs}^{9,10}$ or doping with paramagnetic $\mathrm{Gd}^{3+}$ resulting in $\mathrm{NaGdF}_{4}: \mathrm{Yb}, \mathrm{Er} \mathrm{NPs}^{11,12}$ they have been designed as ultrasmall multimodal agents for tumor imaging. To make dual modal UCNP based probes, in addition to exploiting the insertion of $\mathrm{T} 1$ contrast ions (i.e., $\mathrm{Gd}^{3+}$ ) into the $\mathrm{NaYF}_{4}$ matrix, nanocomposites that combine luminescent UCNPs and MNPs are attracting attention. ${ }^{13,14}$ This results in hybrid probes that combine the efficacy of the UCNPs as an optical imaging probe with the potential applications of MNPs, not only as T2 contrast agents for MRI, but also for cell sorting

applications and as therapeutic agents (i.e., heat mediators in magnetic hyperthermia). Different strategies have been exploited for the preparation of magnetic luminescent nanocomposites based on UCNPs and MNPs. Among them, the cross-linking of MNPs to UCNPs and encapsulation strategies are the most exploited ones. ${ }^{15}$ In addition to the encapsulation strategy using an inorganic shell such as silica beads, polymerbased clustering is an interesting strategy to combine different NPs without altering their magnetic and luminescent properties, resulting in water-dispersible and nontoxic nanomaterials. ${ }^{16}$ Furthermore, encapsulation in polymer enables easy surface functionalization of the resulting nanoclusters to improve cellular uptake and specific recognition, among others. This was demonstrated for instance by wrapping quantum dots (QDs) and MNPs within polymeric beads with a size of few hundreds of nanometers decorated with folic acid molecules. ${ }^{17}$

Received: July 24, 2017

Revised: September 19, 2017

Published: September 25, 2017 


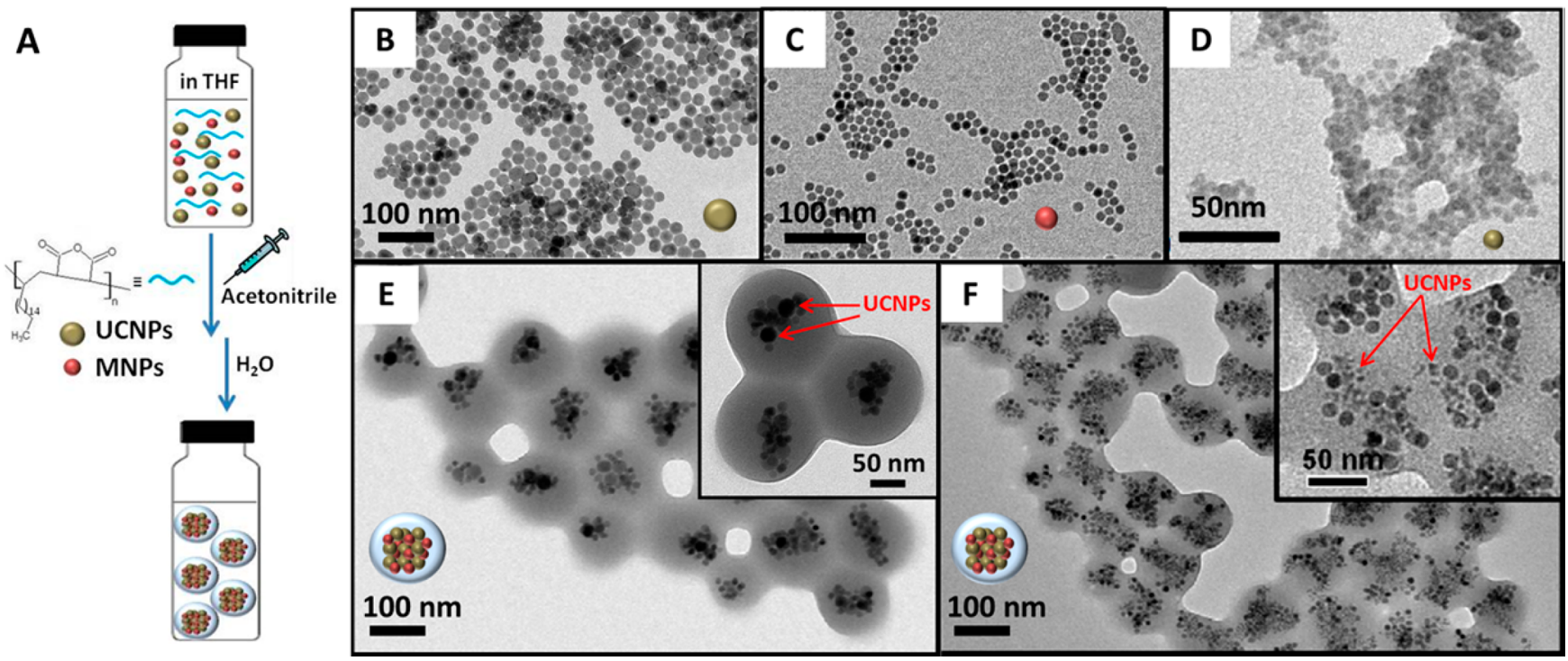

Figure 1. Scheme for synthesis of MUCNBs (A); TEM images of $23 \mathrm{~nm}$ UCNPs (B), $15 \mathrm{~nm}$ IONPs (C), $6 \mathrm{~nm}$ UCNPs (D), and MUCNBs with 15 $\mathrm{nm}$ IONPs and $23 \mathrm{~nm}$ UCNPs (E) or $6 \mathrm{~nm}$ UCNPs (F).

In this work we present the preparation of water-soluble multimodal imaging nanocomposites based on superparamagnetic iron oxide NPs (IONPs) and two different sizes of lanthanide-doped upconverting nanocrystals. The simultaneous incorporation of both NPs was done by wrapping them in the amphiphilic poly(maleic anhydride-alt-1-octadecene), a commercially available polymer, to form a nanobead structure. The resulting clusters exhibit a tunable size between 80 and $130 \mathrm{~nm}$ depending mainly on the NPs and polymer concentrations. We proved that upconversion luminescence and magnetic contrast were still present in the water-soluble hybrid nanocomposites. Moreover, the light emitted by the nanobeads upon NIR excitation was observed through different thicknesses of agarose gel and also through a mouse skin layer simulating an in vivo imaging experiment. In comparison, fluorescent magnetic nanobeads based on quantum dots emitting light in the visible region exhibit significant background autofluorescence from the skin, thus demonstrating the higher efficacy of our nanobeads as in vivo multimodal imaging sensors.

\section{RESULTS AND DISCUSSION}

Preparation of Magnetic Upconverting Nanobeads (MUCNBs). 15-nm-sized superparamagnetic IONPs (Figure 1C) were used as magnetic nanomaterials for the nanobead preparation, and two different upconverting nanocrystals of 23 and $7 \mathrm{~nm}$ (Figure $1 \mathrm{~B}$ and $\mathrm{D}$, respectively) were chosen as luminescent materials. These UCNPs have been demonstrated by Ostrowski et al. ${ }^{18}$ to emit at higher energies in the visible range without sacrificing brightness or stability. To prepare MUCNBs, $15 \mathrm{~nm}$ IONPs and UCNPs (7 or $23 \mathrm{~nm}$ ) were mixed in THF with the presence of the poly(maleic anhydridealt-1-octadecene). The mixture was shaken for $45 \mathrm{~min}$ at room temperature. During that time, the solvent slowly evaporated, allowing the initial packing of particles and polymer. At that point, the controlled addition of a destabilizing solvent like acetonitrile permitted the clustering of IONPs and UCNPs together and the almost simultaneous encapsulation by the polymer, thus resulting in the formation of polymeric beads containing magnetic and UCNPs (Figure 1A). To remove the excess of polymer, the as-prepared MUCNBs were placed on a permanent magnet $(0.3 \mathrm{~T})$ overnight. The supernatant was removed and the solid black pellet containing the nanobeads was collected to the magnet and resuspended in water. After the purification process, TEM images showed the presence inside the beads of both magnetic and UCNPs, which are distinguishable given their different sizes (Figure 1E,F). From these images the MUCNBs exhibit high homogeneity in both size and nanoparticle distribution. The size of the nanobeads and the nanoparticle distribution inside the beads depend on several factors such as nanoparticle and polymer concentration, shaking time, and the rate of acetonitrile addition. These conditions were previously optimized for the preparation of magnetic nanobeads only containing IONPs and resulting in bead sizes between 80 and $200 \mathrm{~nm}^{19}$ Preparation of homogeneous multimodal nanobeads based on different kinds of NPs was conducted by modifying the amount of NPs and keeping the other parameters constant. By mixing $15 \mathrm{~nm}$ IONPs and $7 \mathrm{~nm}$ UCNPs, MUCNBs with hydrodynamic sizes around $120 \mathrm{~nm}$ were obtained. By tuning the nanoparticle concentration the bead size increased from 80 to $120 \mathrm{~nm}$ with a similar distribution of NPs inside the nanobeads, as characterized by TEM (see Figure $\mathrm{S} 1$ in the Supporting Information). For the preparation of the MUCNBs based on 15 $\mathrm{nm}$ IONPs and $23 \mathrm{~nm}$ UCNPs, the same trend was observed. Larger hydrodynamic sizes ranging from 150 to $160 \mathrm{~nm}$ in ACN were observed immediately after their preparation. Diameters of 160 to $170 \mathrm{~nm}$ were observed after being transferred into water (Figures S2 and S3). From dynamic light scattering (DLS) spectra (Figures S3 and Table S1), narrow peaks are recorded both in $\mathrm{ACN}$ and in water, indicating the absence of aggregates for the MUCNBs and demonstrating the monodispersion of the MUCNBs in solution. The slight increase in the hydrodynamic diameter upon transfer from ACN to water suggests hydration of the polymer shell.

The nanoparticle distribution differed slightly in the nanobeads containing 23 or $7 \mathrm{~nm}$ UCNPs. The TEM image shows a clustering of few $23 \mathrm{~nm}$ UCNPs within the MUCNBs compared to the $7 \mathrm{~nm}$ UCNPs which in the latter case formed larger clusters inside the nanobeads and were more uniformly distributed with IONPs (Figure 1E,F). This could be due to a 


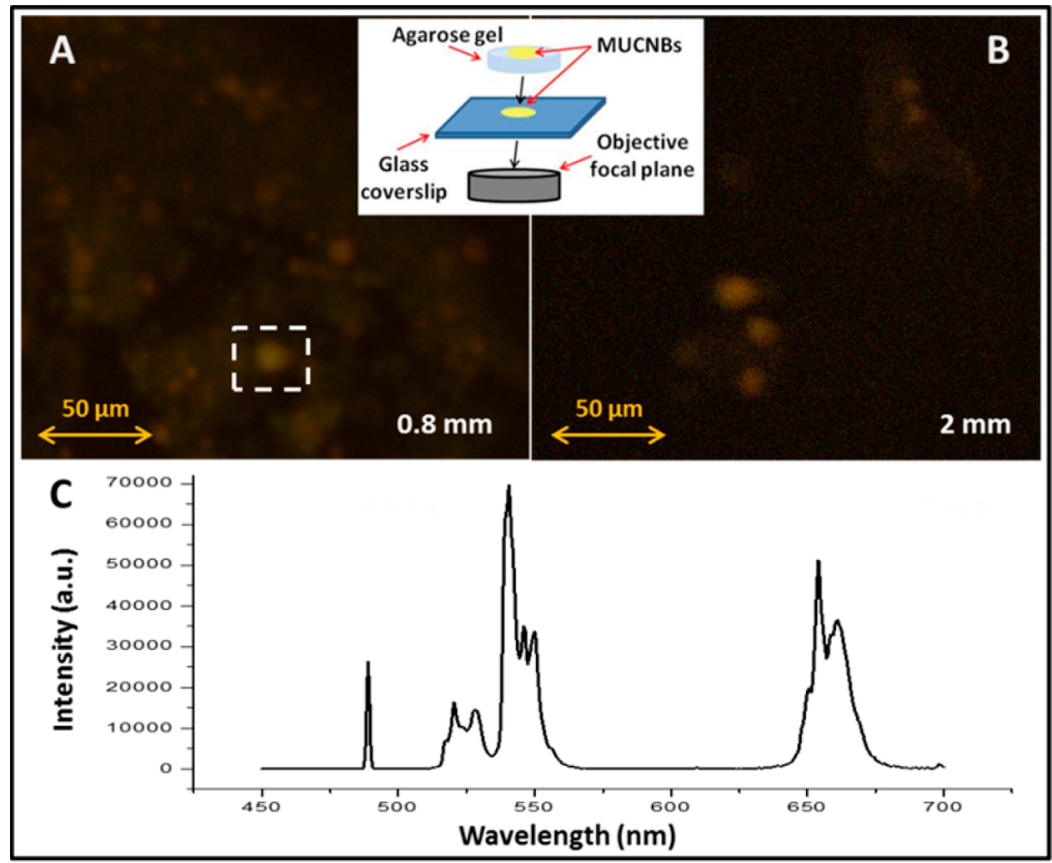

Figure 2. Schematic representation of a confocal experiment performed on MUCNBs with 23 nmUCNPs deposited on agarose gel. Confocal spectral image of MUCNBs with $23 \mathrm{~nm}$ UCNPs deposited on an agarose gel of $0.8 \mathrm{~mm}$ thickness (A) and $2 \mathrm{~mm}$ thickness (B). Emission spectrum of MUCNBs with $23 \mathrm{~nm}$ UCNPs $\left(\lambda_{\text {exc }}=980 \mathrm{~nm}\right)(\mathrm{C})$.

difference in the destabilization of the larger UCNPs with respect to the smaller MNPs. To deeply investigate this aspect, MUCNBs were also prepared using larger UCNPs (30-40 $\mathrm{nm})$ and small manganese iron oxide NPs $(8 \mathrm{~nm})$. TEM images of these MUCNBs have indeed shown large clusters of manganese iron oxide NPs and mostly only 2-3 UCNPs per bead (Figure S4) thus confirming the more difficult destabilization of the bigger UCNPs with respect to the smaller MNPs. In this specific case, however, given the high packing density of MNPs around each UCNP within the beads the photoluminescence was not retained (data not shown).

Confocal Microscopy. To further confirm the success of the co-clustering of both IONPs and UCNPs in the nanobead structure and also to verify the retention of the UCNPs' upconverting luminescence, the MUCNBs were analyzed by optical spectroscopy, lifetime analysis and confocal microscopy. Under near-infrared excitation (980 nm, $2 \mathrm{~W})$, MUCNBs retained the green $(520,540 \mathrm{~nm})$ and red luminescence $(653$ $\mathrm{nm}$ ) peaks characteristic of the $\mathrm{Yb}^{3+} / \mathrm{Er}^{3+}-\mathrm{NaYF}_{4} \mathrm{UCNPs}$. The $653 \mathrm{~nm}$ emission of the MUCNBs exhibited an effective luminescence lifetime of $214.2 \pm 0.9 \mu \mathrm{s}$, a factor of 2 lower than the $478 \pm 5 \mu$ s lifetimes of the nanobeads loaded with only UCNPs (see Figure S5 and Table S2 in SI for detailed lifetime data). This reduction in the effective lifetime is due to the introduction of a second exponential decay component (130 $\mu \mathrm{s}$ ) that may represent the nonradiative transfer of energy from UCNP excited states to adjacent IONPs. Similarly, the green emission (540 nm) of MUCNBs exhibits a luminescence lifetime that is 2 -fold shorter than beads with only UCNPs.

Although the reduced lifetime at both wavelengths implies a reduction in upconversion efficiency, the fact that the lifetimes are not reduced more significantly allows the MUCNBs to retain their ability to upconvert NIR light. In fact, typical MUCNB suspensions were consistently brighter than those without IONPs because isolation of the magnetic beads from their reaction solutions was more straightforward.
When drop-cast on a glass coverslip and illuminated under near-infrared excitation ( $980 \mathrm{~nm})$, MUCNBs containing both sizes of UCNPs exhibited bright emission intensity in the visible region of 500-680 $\mathrm{nm}$ (Figure S6). However, the efficiency of the MUCNBs as potential light sensors depends on the capacity of these luminescent materials to emit visible light through different tissues without increasing the corresponding background from the tissues which slightly absorb NIR light. ${ }^{8,20,21}$ Therefore, the MUCNBs were analyzed through different matrixes and thicknesses to emulate the in vivo conditions. For instance, the MUCNBs deposited on a glass coverslip were covered with agarose gels of different thicknesses $(0.8,2.0$, and $4.0 \mathrm{~mm})$ and then drop-cast again on top of the gel to study the light penetration. The beads on the coverslip were used as a reference to focus the confocal image at the gel base, then images were collected by scanning the sample along the $z$ axis ( $z$-stack) until the top surface of the gel was reached. In Figure S7A, the 3D confocal image recorded for the agarose gel of $0.8 \mathrm{~mm}$ thickness is reported. As shown in Figure $2 \mathrm{~A}$ and $\mathrm{B}$, the luminescent nanobeads were clearly observed as yellow spots respectively at 0.8 and $2.0 \mathrm{~mm}$ of thickness upon excitation at $980 \mathrm{~nm}$ (Figure S7). These yellow spots correspond to the MUCNBs as confirmed by the emission spectra recorded in those regions which present typical emission peaks of NaYF4:Yb, ErNPs at 520, 540, and $650 \mathrm{~nm}$ (Figure 2C). Nevertheless, a further increase of the gel thickness to $4 \mathrm{~mm}$ resulted in poor detection of the nanobeads (Figure S7B). Therefore, the observed intensity of MUCNBs upon NIR excitation is directly related to the thickness of the agarose gel, as a decrease of the emission intensity is observed with an increasing thickness. However, a high emission intensity at a very high depth is desirable for cell and tissue imaging. An excitation at $970 \mathrm{~nm}$ with a pulsed laser was applied through different thicknesses of agarose gel up to 5 $\mathrm{mm}$. In this case, the MUCNBs were clearly observed as bright spots on the top surface of the gel (Figure S8) even through a 
thickness of $5 \mathrm{~mm}$. Indeed, the stronger excitation power ensured by the pulsed laser (500 $\mathrm{mW}$ mean power) with respect to the previously used continuous emitting laser (150 $\mathrm{mW}$ ) enhances the imaging efficiency of the MUCNBs. Based on these results, we sought to evaluate the efficiency of these new luminescent nanoclusters as imaging sensors through a tissue representative of human skin-mouse skin. For this purpose, we followed a procedure similar to the one reported in Figure 2 but substituting the agarose gel with a slice of hairless mouse skin with a thickness of $0.5 \mathrm{~mm}$. It is well-known that autofluorescence from the skin interferes in the visualization of the clusters, thereby decreasing the signal-to-noise ratio and imaging sensitivity. The main issue arises from the visible light absorption by the skin, which is negligible in the NIR range. In fact, the MUCNBs were readily visible through the mouse skin with a high contrast under NIR excitation, without interference from the background fluorescence from the skin (Figure 3A).

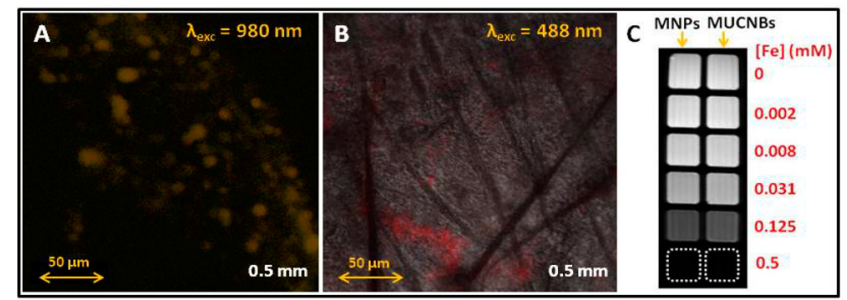

Figure 3. Confocal images of MUCNBs with UCNPs of $23 \mathrm{~nm}$, deposited on a slice of mouse skin (thickness $0.5 \mathrm{~mm}, \lambda_{\mathrm{exc}}=980 \mathrm{~nm}$, $\lambda_{\mathrm{em}}=500-680 \mathrm{~nm}$ ) (A). Confocal images of MFNBs deposited on a slice of mouse skin (thickness $0.5 \mathrm{~mm}, \lambda_{\mathrm{exc}}=480 \mathrm{~nm}, \lambda_{\mathrm{em}}=640 \mathrm{~nm}$ (B); T2-weighted MR image of magnetic nanoparticles (MNPs) and magnetic upconverting nanobeads (MUCNBs) at different concentrations $(\mathrm{C})$.

In contrast, the MFNBs based on quantum dots (488 nm excitation, $600 \mathrm{~nm}$ emission) and imaged under the same conditions exhibited a high background noise related to the autofluorescence of the skin, thus reducing the effectiveness of the QDs as potential in vivo sensors (Figure 3B). Indeed, the excitation of the mouse skin at the corresponding wavelength of the MFNBs (488 nm) resulted in autofluorescence in the visible emission range $(520-700 \mathrm{~nm})$, in which the QDs also emit (Figure S9A). In contrast, the excitation of the mouse skin at the MUCNBs excitation wavelength $(980 \mathrm{~nm})$ produced no emission in the visible range (Figure S9B), thus indicating a high signal to the noise ratio upon NIR excitation of the MUCNBs in medical imaging techniques.

Relaxivity Measurements. As commented above, the inclusion of both upconverting and iron oxide NPs in the same nanobead enables the exploitation of the synthesized MUCNBs as multifunctional imaging sensors. Having demonstrated their potential application as imaging probes upon NIR excitation, the MUCNBs were then evaluated as magnetic resonance imaging $^{22}$ contrast agents by performing relaxivity measurments.

The IONPs used for the bead preparation have been shown to have high values for the transverse relaxation rates $r_{2}$ making them efficient negative contrast agents for MRI. ${ }^{23}$ To evaluate if the clustering process affects the relaxation times of the magnetic NPs, the $r_{2}$ values measured for the MUCNBs were compared with those of the single $15 \mathrm{~nm}$ IONPs. The measurements were performed at two different magnetic fields (1.5 and 9.4 T). The IONPs were transferred to water as the
NPs were individually coated with the same poly(maleic anhydride-alt-1-octadecene) used in the MUCNB's formation.

Similar $r_{2}$ values were obtained for both MUCNBs and IONPs. At a low magnetic field, $1.5 \mathrm{~T}$, the IONPs presented an $r_{2}$ value of $112 \mathrm{mM}^{-1} \mathrm{~s}^{-1}$, while the MUCNBs exhibited an $r_{2}$ value of $122 \mathrm{mM}^{-1} \mathrm{~s}^{-1}$. At a high magnetic field, $9.4 \mathrm{~T}, r_{2}$ values of 208 and $206 \mathrm{mM}^{-1} \mathrm{~s}^{-1}$ for single IONPs and MUCNBs were obtained, respectively (see also Table S3 in SI). These transverse relaxivity results are in accordance with the values reported in the literature for individual and clustered IONPs with sizes similar to those of our nanocomposites. ${ }^{24-26}$ The similar capacity of both IONPs and MUCNBs to act as T2 contrast agent for MRI was also qualitatively observed by imaging the two materials in a multiwell phantom for MRI in which the two samples were drop-cast at different iron concentrations. Indeed, as shown in Figure 3C, IONCs and MUCNBs exhibit similar signal decay, that is, similar T2 values, at the same iron concentration.

Cytotoxicity Assay and Stability in Cell Media. A lactate dehydrogenase $(\mathrm{LDH})$ membrane integrity assay was performed to measure the cytotoxicity of MUCNBs at different incubation times (12 and $24 \mathrm{~h}$ ) and with different doses. Doses were based on the elemental analysis of iron $(6,12$, and $24 \mu \mathrm{g}$ $\mathrm{Fe} / \mathrm{mL})$ and the corresponding amounts of yttrium $(2.58,5.17$, and $10.33 \mu \mathrm{g}_{\mathrm{Y}} / \mathrm{mL}$ ) administered to two cancer cell lines (HeLa-WT cervical cancer cells and A431 epidermoid carcinoma cells). Within the concentration range chosen, HeLa-WT cervical cancer cells did not show any sign of cytotoxicity ( $<5 \%$ toxicity) after 12 and $24 \mathrm{~h}$ of incubation (Figure 4a). A similar trend was also observed when using A431 cells and testing the toxicity at $12 \mathrm{~h}$; a slightly higher cytotoxicity (ca. 25\%) was observed at $24 \mathrm{~h}$ (Figure $4 \mathrm{~b}$ ). We also examined the stability of MUCNBs in the cell culture media by observing sedimentation at $37^{\circ} \mathrm{C}$. No sedimentation

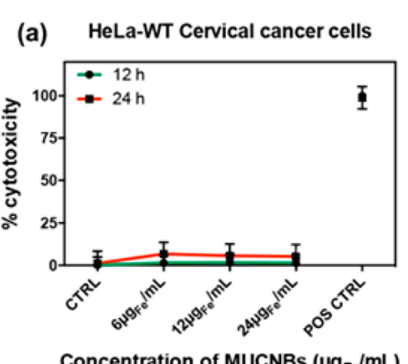

(c)

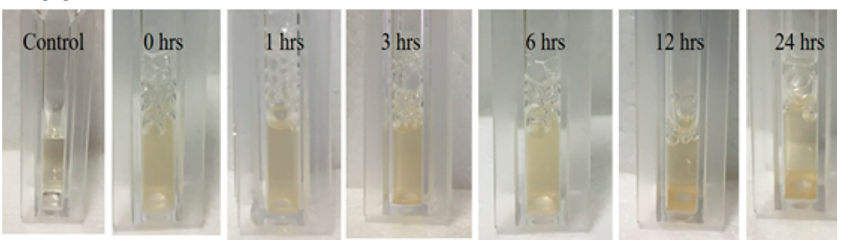

Figure 4. LDH assay showing the percentage of cytotoxicity as a function of the concentration of MUCNBs for HeLa-WT cells, showing no toxicity at 12 and $24 \mathrm{~h}$ (a) and for A431 cells, showing a slight toxicity $(25 \%)$ at $24 \mathrm{~h}$ and no toxicity at $12 \mathrm{~h}(\mathrm{~b})$. Error bars represent the standard deviation ( $n=3$ for all groups). Optical images (c) of MUCNBs $(6 \mu \mathrm{g} \mathrm{Fe} / \mathrm{mL})$ at $0,1,3,6,12,24 \mathrm{~h}$ of incubation in phenyl red free DMEM medium containing $10 \%$ of FBS. The first panel (control) represents plain DMEM. MUCNBs remain stable in media for up to $6 \mathrm{~h}$ of incubation, after which the bead sample began to partially sediment. 
was observed for up to $6 \mathrm{~h}$ of incubation, while partial sample sedimentation was observed at 12 and $24 \mathrm{~h}$ (Figure 4c). The hydrodynamic diameters measured in water just after the exposure of the MUCNBs to cell culture media indicated an increase in size when prolonging the exposure time in the media (Figure S10). This size increase can likely be attributed to the partial absorption of serum proteins that indeed bring to the partial beads sedimentation and in turn might account for the slight toxicity observed with the A431 cells at $24 \mathrm{~h}$ (Figure $4 \mathrm{~b})$. Overall, the trend in the cytotoxicity of the MUCNBs is consistent with that of magnetic nanobeads that contain only iron oxide NPs, with the same dose ranges and incubation times, as we have previously demonstrated (ESI of the work of R. Di Corato et al.). ${ }^{27}$

\section{CONCLUSIONS}

In this work, we have reported the fabrication of water-soluble nanobeads with both magnetic and upconverting functionality. The nanobeads were prepared by combining $15 \mathrm{~nm}$ iron oxide NPs with NaYF4:Yb,ErNPs (UCNPs) of two different sizes (23 and $7 \mathrm{~nm}$ ) wrapped together in the amphiphilic poly(maleic anhydride-alt-1-octadecene). Depending on the concentration of NPs and polymer, the nanobead size was tunable from 80 to $160 \mathrm{~nm}$. These MUCNBs were successfully imaged through different thicknesses and matrixes upon a near-infrared excitation wavelength at $980 \mathrm{~nm}$. In comparison to other fluorescent nanobeads based on QDs, the upconverting nanobeads did not show the autofluorescent background of the skin due to the NIR laser as excitation source. Moreover, these MUCNBs were demonstrated to be outstanding T2 contrast agents at low and high magnetic fields. The facile synthesis and ready functionalization of these multifunctional magnetic and upconverting nanobeads will allow them to be used in a range of in vivo probes for molecular imaging. Finally, no significant toxicity was observed for the MUCNBs. The resulting system can be easily used as a starting platform for further modification to obtain more complex hybrid systems.

\section{EXPERIMENTAL SECTION}

Chemicals. Poly(maleic anhydride-alt-1-octadecene), Mn $30000-50000 \mathrm{~g} / \mathrm{mol}$ (Aldrich), Milli-Q water (18.2 M , filtered with filter pore size $0.22 \mu \mathrm{M}$ ) from Millipore, acetonitrile (HPLC grade, J. T. Baker) and tetrahydrofuran anhydride (Carlo Erba, p.a.), iron oxide hydroxide (SigmaAldrich, \#371254), iron acetylacetonate (Sigma-Aldrich, 99\%), manganese acetylacetonate (Sigma-Aldrich, \#245763), hexadecanediol (Sigma-Aldrich, 90\%), dodecylamine (Sigma-Aldrich, 98\%), lauric acid (Sigma-Aldrich, 99\%), benzyl ether (SigmaAldrich, 98\%), and octadecene (Sigma-Aldrich, 90\%), $\mathrm{YCl}_{3}$, $\mathrm{YbCl}_{3}, \mathrm{ErCl}_{3}$, ammonium fluoride, and sodium oleate, tri- $n$ octylphosphine oxide (Strem, 99\%), tri- $n$-octylphosphine (Strem, 97\%), n-octadecylphosphonic acid (PolyCarbon Industries, 99\%), cadmium oxide (Sigma-Aldrich, 99.99\%), selenium (Sigma-Aldrich, 99.99\%), diethylzinc (Sigma-Aldrich, $1 \mathrm{M}$ in hexane) and hexamethyldisilathiane (Sigma-Aldrich, synthesis grade), ethanol (Sigma-Aldrich, 99.8\%), methanol (Sigma-Aldrich, 99.9\%), toluene (Sigma-Aldrich, 99.5\%), and hexane (Sigma-Aldrich, 99\%) were used without any further purification. All reagents for cell cytotoxicity assay were purchased from Gibco (ThermoFisher Scientific).

Synthesis of 15-nm-size Iron Oxide Nanoparticles (IONPs). IONPs were prepared following the method published by Colvin et al. ${ }^{28}$ Briefly, $0.18 \mathrm{~g}$ of iron oxide hydroxide, 2.3 or $3.45 \mathrm{~g}$ of oleic acid (leading to a ratio of oleic acid to iron oxide hydroxide of 4 or 6 , respectively), and $5 \mathrm{~g}$ of octadecene were transferred in a three-necked round-bottom flask to an under inert gas atmosphere and stirred at $320^{\circ} \mathrm{C}$ for $1 \mathrm{~h}$. Finally, the NPs were washed twice by the addition of ethanol or methanol as nonsolvents followed by centrifugation. The supernatant was removed and the particles were redispersed in toluene.

Synthesis of Upconverting $\mathrm{NaYF}_{4}$ Nanoparticles Doped with $\mathrm{Yb}^{3+}$ and $\mathrm{Er}^{3+}$ (UCNPs). $\mathrm{NaYF}_{4}: 20 \% \mathrm{Yb}^{3+}, 2 \%$ $\mathrm{Er}^{3+} \mathrm{NPs}$ were synthesized in an automated nanoparticle synthesis robot (WANDA) using the method described by Ostrowski et al. ${ }^{18}$ Briefly, a stock solution of rare earth oleates (78 mol \% Y, 20\% Yb, 2\% Er) was prepared by heating $\mathrm{YCl}_{3}$, $\mathrm{YbCl}_{3}, \mathrm{ErCl}_{3}$, and oleic acid in 1-octadecene under vacuum. Reactions were performed under nitrogen in WANDA by adding the stock solution, ammonium fluoride, sodium oleate to a $40 \mathrm{~mL}$ glass vial and heating the vial to $305-318^{\circ} \mathrm{C}$ for 45 min while stirring. The nanoparticle product was purified from reactants using three cycles of precipitation with ethanol, centrifuging, and resuspension in hexane. To grow smaller 8 nm UCNPs, oleylamine was added to the final reaction solution. $^{18}$

Synthesis of 8-nm-size Manganese Iron Oxide Nanoparticles. Manganese iron oxide NPs were prepared by following a previous paper. ${ }^{29} 2 \mathrm{mmol}$ of iron acetylacetonate was mixed with $1 \mathrm{mmol}$ manganese acetylacetonate, $10 \mathrm{mmol}$ hexadecanediol, $6 \mathrm{mmol}$ dodecylamine, $6 \mathrm{mmol}$ lauric acid, and $20 \mathrm{~mL}$ benzyl ether. The solution was exposed to a flow of nitrogen and heated to $140{ }^{\circ} \mathrm{C}$ for $1 \mathrm{~h}$, then to $210{ }^{\circ} \mathrm{C}$ for $2 \mathrm{~h}$, and finally to $300{ }^{\circ} \mathrm{C}$ for another $1 \mathrm{~h}$. Subsequently, the sample was washed several times with ethanol, acetone, and isopropanol as precipitation agents, then centrifuged. The supernatant was removed and the particles redispersed in toluene.

Synthesis of 6-nm-size Cadmium Selenide-Zinc Sulfide Core-Shell Quantum Dots (QDs). Cadmium selenide-zinc sulfide QDs were prepared in a two-step process according to some modifications of already published protocols. $^{30,31}$ Nearly monodisperse $5 \mathrm{~nm}$ CdSe QDs were synthesized by mixing them in a $50 \mathrm{~mL}$ flask with $3.0 \mathrm{~g}$ of trioctylphosphine oxide, ${ }^{32} 0.280 \mathrm{~g}$ of $n$-octadecylphosphonic acid (ODPA), and $0.060 \mathrm{~g}$ of cadmium oxide. ${ }^{33}$ The reaction mixture was heated to ca. $150{ }^{\circ} \mathrm{C}$ and exposed to vacuum for ca. $1 \mathrm{~h}$. Then, under nitrogen atmosphere, the solution was heated to above $300{ }^{\circ} \mathrm{C}$ to dissolve the $\mathrm{CdO}$ until it turned optically clear and colorless. At this point, $1.5 \mathrm{~g}$ of TOP was injected into the flask and the temperature was increased to $380{ }^{\circ} \mathrm{C}$ for the injection of the Se:TOP solution ( $0.058 \mathrm{~g} \mathrm{Se}+0.360 \mathrm{~g}$ TOP). The reaction was stopped after $1 \mathrm{~min}$ to synthesize the red fluorescent CdSe QDs. To form the $\mathrm{ZnS}$ shell, the temperature of the reaction was decreased to $120{ }^{\circ} \mathrm{C}$ and a solution of 450 $\mu \mathrm{L}$ of diethylzinc $\left(\mathrm{ZnEt}_{2}\right)$ and $125 \mu \mathrm{L}$ of hexamethyldisilathiane ((TMS $\left.)_{2} \mathrm{~S}\right)$ dissolved in $1.5 \mathrm{~mL}$ of TOP was injected dropwise into the reaction flask.

The temperature was then increased to $200{ }^{\circ} \mathrm{C}$ for $10 \mathrm{~min}$ under stirring. The mixture was cooled down to $90{ }^{\circ} \mathrm{C}$ and 3 $\mathrm{mL}$ of toluene were added. After the synthesis, the nanocrystals were washed by repeated dissolution in toluene and precipitation with the addition of methanol and, finally, they were dissolved in toluene. 
Synthesis of Magnetic Upconverting Nanobeads (MUCNBs) with UCNPs of 23 and $7 \mathrm{~nm}$. The synthesis of MUCNBs with UCNPs is based on a modified procedure reported here for the synthesis of MFNBs with QDs. ${ }^{17} 6.2 \mu \mathrm{L}$ of IONPs ${ }^{28}$ in chloroform (0.27 $\mu \mathrm{M}$ NPs concentration, $d=15$ $\pm 1 \mathrm{~nm})$ and $5 \mu \mathrm{L}$ of $23 \pm 1 \mathrm{~nm} \mathrm{UCNPs} \mathrm{in} \mathrm{hexane} \mathrm{or} 10 \mu \mathrm{L}$ of $7 \pm 1 \mathrm{~nm}$ UCNPs in toluene (yttrium concentration was $3.1 \mathrm{~g}$ / $\mathrm{L}$ for the $23 \mathrm{~nm}$ particles and $1.8 \mathrm{~g} / \mathrm{L}$ for the $7 \mathrm{~nm}$ particles) were transferred in an $8 \mathrm{~mL}$ open vial. The solvent was evaporated by fluxing nitrogen and the particles were redispersed in $185 \mu \mathrm{L}$ of THF. $15 \mu \mathrm{L}$ of poly(maleic anhydride-alt-1-octadecene) in THF (50 mM, this concentration refers to the polymer monomer units) was added and the mixture was shaken for $45 \mathrm{~min}$ (1000 rpm shaking rate), keeping the vial open. Then, $0.8 \mathrm{~mL}$ of acetonitrile was added dropwise at a flow rate of $250 \mu \mathrm{L} / \mathrm{min}$. After the bead formation, the sample was cleaned by placing it on top of a permanent magnet $(0.3 \mathrm{~T})$ overnight followed by the redispersion of the nanobeads collected to the magnet in 500 $\mu \mathrm{L}$ of fresh water.

Synthesis of Magnetic Fluorescent Nanobeads (MFNBs) with QDs. The synthesis of MFNBs with QDs is based on a previous reported work. ${ }^{17} 7 \mu \mathrm{L}$ of manganese iron oxide $\mathrm{NPs}^{29}$ in toluene $(36.76 \mu \mathrm{M}$ NPs concentration, $d=8 \pm$ $1 \mathrm{~nm})$ and $8.64 \mu \mathrm{L}$ of cadmium selenide-zinc sulfide core-shell $\mathrm{QDs}^{31}$ in toluene (40 $\mu \mathrm{M}$ NPs concentration, $\left.d=6 \pm 1 \mathrm{~nm}\right)$ were put in an $8 \mathrm{~mL}$ open vial. The solvent was evaporated by fluxing nitrogen and the particles were redispersed in $175 \mu \mathrm{L}$ of THF. $25 \mu \mathrm{L}$ of poly(maleic anhydride-alt-1-octadecene) in THF ( $50 \mathrm{mM}$, this concentration refers to the polymer monomer unit) were added and the mixture was shaken for 45 min (1000 rpm shaking rate). Then, $0.8 \mathrm{~mL}$ of acetonitrile was added dropwise at a flow rate of $250 \mu \mathrm{L} / \mathrm{min}$. After the nanobeads' formation, the sample was cleaned initially by placing it onto a permanent magnet $(0.3 \mathrm{~T})$ for $10 \mathrm{~min}$ to remove the biggest beads, and then the supernatant was cleaned again on the magnet overnight, followed by the redispersion of the beads in $500 \mu \mathrm{L}$ of fresh water.

Cell Culture. A431 epidermoid carcinoma and HeLa-WT cervical cancer cell lines were cultured using Dulbecco's modified eagle's medium (DMEM) supplemented with 10\% fetal bovine serum (FBS), 2\% Penstrep, and 1\% L-Glutamine at $37{ }^{\circ} \mathrm{C}$ and $5 \% \mathrm{CO}_{2}$. The cells were grown to confluency in $\mathrm{T} 75$ cell culture flask.

Administration of MUCNBs and LDH Cytotoxicity Assay. To measure the toxicity of the MUCNBs, both cell types were washed using PBS, trypisized using Trypsin/EDTA (0.025/0.01\% in PBS), counted using NucleoCounter NC$100^{\mathrm{TM}}$ (Chemometec), and plated in 24-well plates at a density of 100000 cells/well for 12 and $24 \mathrm{~h}$ time points. The cells were then allowed to grow overnight in an incubator at $37^{\circ} \mathrm{C}$. The next day the media on the cells was replaced with $500 \mu \mathrm{L}$ of 6,12 , or $24 \mu \mathrm{g}_{\mathrm{Fe}} / \mathrm{mL}$ of MUCNBs per well, prepared by diluting a stock solution of MUCNBs in water $\left(1.51 \mathrm{mg}_{\mathrm{Fe}} / \mathrm{mL}\right.$, $0.65 \mathrm{mg}_{\mathrm{Y}} / \mathrm{mL}$ ) in phenol-red free complete media. The use of phenol-red free media ensured that the phenol-red indicator would not interfere with our assays. The cell plates were then placed in the sterile incubator at $37{ }^{\circ} \mathrm{C}$ with $5 \% \mathrm{CO}_{2}$ and allowed to grow for 12 and $24 \mathrm{~h}$ before carrying out the toxicity assay. Separate wells were allocated for control experiments in which no NPs were added (see later CTRL and POS CTRL). Prior to starting the assay, a positive control (POS CTRL, $100 \%$ toxicity) was prepared. $50 \mu \mathrm{L}$ of lysis buffer provided by the kit manufacturer was added to the cell wells allocated for the positive controls (POS CTRL) and incubated at $37{ }^{\circ} \mathrm{C}$ with $5 \% \mathrm{CO}_{2}$ for $45 \mathrm{~min}$. A negative control (CTRL) consists of cells that was grown under the same conditions without treatment with MUCNBs and with no disturbance. After the respective time points, the cytotoxicity of the MUCNBs was assessed using a Pierce LDH cytotoxicity assay kit (ThemoFisher Scientific). This assay was performed three times for each condition. Later, the supernatant from each well (CTRL, POS CTRL, 6, 12, and $24 \mu \mathrm{g}_{\mathrm{Fe}} / \mathrm{mL}$ UCNPs) was collected in an Eppendorf tube and centrifuged at $250 \mathrm{~g}$ for $3 \mathrm{~min}$. $50 \mu \mathrm{L}$ aliquots of these supernatants were then transferred to a clean 96 well plate, and $50 \mu \mathrm{L}$ of $\mathrm{LDH}$ assay mix was added to each well. The 96-well plate was then incubated at room temperature in the dark for $30 \mathrm{~min}$. Finally, after the incubation period, 50 $\mu \mathrm{L}$ of stop solution that was in the kit was added to each well and absorbance was read at 490 and $680 \mathrm{~nm}$ (for background correction) using a MultiSkan Go Multiplate Spectometer (ThermoFisher Scientific). The percent toxicity was then calculated using the formula given below after blank subtraction and the mean values were plotted with respective error bars. ${ }^{26}$ Each data point was repeated three times for all groups $(n=3)$.

$$
\begin{aligned}
\text { \%toxicity }= & (\text { experimental Abs value }- \text { MUCNBs Abs value } \\
& - \text { CTRL Abs value }) /(\text { POS CTRL Abs value } \\
& - \text { CTRL Abs value }) \times 100
\end{aligned}
$$

in which Experimental Abs value is the absorption value of each condition tested; MUCNBs Abs value is the absorption value of $24 \mu \mathrm{gFe} / \mathrm{mL}$ MUCNBs in cell media; CTRL Abs value is the absorption value of the untreated cells; POS CTRL Abs value is the absorption value of the positive control cells.

Stability of MUCNBs in Cell Media. To evaluate the stability of MUCNBs in cell culture media, $15 \mu \mathrm{L}$ of MUCNBs $\left(0.4 \mathrm{mg}_{\mathrm{Fe}} / \mathrm{mL}\right)$ was added to $500 \mu \mathrm{L}$ of phenyl-red free DMEM medium containing $10 \%$ fetal bovine serum (FBS), and incubated for an additional 6, 12, and $24 \mathrm{~h}$, at $37^{\circ} \mathrm{C}$. After the incubation periods, the MUCNBs were collected by a permanent magnet $(0.3 \mathrm{~T})$, redispersed in $500 \mu \mathrm{L}$ of Milli-Q water and the average hydrodynamic sizes were determined by DLS (Figure S10a). The MUCNB solutions in water were then left undisturbed for 6 additional hours and DLS measurements were repeated (Figure S10b). DLS hydrodynamic diameters are summarized in Table S4.

Elemental Analysis. An inductively coupled plasma atomic emission spectrometer (ICP-AES, iCAP 6500, Thermo) was used for the elemental analysis and the concentration evaluation of magnetic, fluorescent, and UCNPs. The samples were prepared by an overnight digestion of $25 \mu \mathrm{L}$ of sample solution in $2.5 \mathrm{~mL}$ of aqua regia. Subsequently, the sample was diluted with Milli-Q water to a final volume of $25 \mathrm{~mL}$.

TEM Characterization. Transmission electron microscopy was performed on a JEOL JEM-1011 with an acceleration voltage of $100 \mathrm{kV}$. The sample preparation was conducted by drop-casting a droplet of the sample solution onto a carbon coated copper grid with the subsequent removal of the solvent by evaporation at room temperature.

Dynamic Light Scattering (DLS) Characterization. Dynamic light scattering measurements were performed on a Zetasizer Nano ZS90 (Malvern, USA) equipped with a $4.0 \mathrm{~mW}$ $\mathrm{He}-\mathrm{Ne}$ laser operating at $633 \mathrm{~nm}$ and an avalanche photodiode detector. Measurements were conducted with a ZEN0112-low 
volume disposable sizing cuvette, setting 1.330 as the refractive index and $0.8869 \mathrm{cP}$ as the viscosity. The measurements were performed with 173 backscatter (NIBS default) as angle of detection, with an automatic scan time and three scans per measurement.

Relaxivity Measurements. Water solutions of MFNBs, MUCNBs, and IONPs with iron concentration ranging from 0.002 to $0.5 \mathrm{mM}$ were prepared. The longitudinal $\left(T_{1}\right)$ and transverse $\left(T_{2}\right)$ relaxation times were measured at $37^{\circ} \mathrm{C}$ using a Minispec spectrometer (Bruker, Germany) mq 60 (1.5 T) and a 9.4 T Biospec (Bruker, Germany) system equipped with a 400 $\mathrm{mT} / \mathrm{m}$ gradient and a $40 \mathrm{~mm}$ quadrature bird-cage resonator. The $T_{1}$ relaxation profile was obtained using an inversionrecovery sequence, with 20 data points and 4 acquisitions for each measurement. The $T_{2}$ relaxation time was measured using a Carr-Purcell Meiboom Gill (CPMG) spin-echo pulse sequence with 200 data points with an inter echo time of 0.5 ms. The relaxivities $r_{i}(i=1,2)$ were determined by the following equation:

$$
\frac{1}{T_{i(\mathrm{Obs})}}=\frac{1}{T_{i\left(\mathrm{H}_{2} \mathrm{O}\right)}}+r_{i} \mathrm{C}_{\mathrm{Fe}} \quad(i=1,2)
$$

in which $C_{\mathrm{Fe}}$ is the concentration of $\mathrm{Fe}$ ions. The values are reproducible within a $5 \%$ deviation.

Confocal Microscopy. Confocal microscopy images were acquired by an Al Nikon Eclipse Ti microscope. For the MFNBs, the microscope was equipped with an argon laser source at $488 \mathrm{~nm}$. However, to detect MUCNBs, a fiber coupled CNI laser CW with a power emission of $150 \mathrm{~mW}$ emitting at $980 \mathrm{~nm}$ was used. Images were recorded using an acquisition window set at 525-700 nm for the red emitting QDs and 500-700 nm for the UCNPs. For the analysis, the nanobead samples were deposited by drop casting on a $75 \times 25$ $\mathrm{mm}$ microscope glass coverslip. To compare the efficacy of both fluorescent and upconverting materials as luminescent probes for tissues, confocal microscopy images were taken for both kinds of nanobeads through different materials: agarose gel slices ( $2 \%$ in agarose) of different thickness $(0.8-2$ and 4 $\mathrm{mm})$ and mouse skin ( $0.45 \mathrm{~mm}$ thickness). In these experiments the nanobead solutions were deposited on the glass coverslip, and then the gel layer or the mouse skin was placed on the glass and the nanobead solution was drop-cast again on top of the gel or the mouse skin. Consequently, it was possible to detect the presence of both types of MFNBs and MUCNBs on the glass surface in addition to the top of the different materials with different thicknesses. Under the same conditions of excitation and emission used to detect the two kinds of nanobeads, the different substrates (agarose gel and mouse skin) were also observed alone. Further analyses were conducted on the MFNBs and MUCNBs deposited on agarose gel layers according to the previous procedure, using a twophoton confocal microscope Olympus FV1000MPE equipped with a Ti:sapphire laser Spectra Physics MaiTai emitting at 970 $\mathrm{nm}$ with a pulse of $100 \mathrm{fs}$, a frequency of $80 \mathrm{MHz}$, and a power emission of $500 \mathrm{~mW}$. Steady-state upconversion spectroscopy and lifetime measurements. Steady-state upconversion luminescence spectra and lifetime measurements of UCNPs and MUCNBs were acquired in an Edinburgh FLS980 spectrometer. Solution-phase samples were excited with a $2 \mathrm{~W}, 980 \mathrm{~nm}$ laser. Time-dependent lifetime decays were initiated with nanosecond laser pulses from the $980 \mathrm{~nm}$ laser and were measured using the multichannel scaling (MCS) technique.

\section{ASSOCIATED CONTENT}

\section{Supporting Information}

The Supporting Information is available free of charge on the ACS Publications website at DOI: 10.1021/acs.bioconjchem.7b00432.

Additional TEM characterizations and confocal images, PL spectra and time dependent decay measurements (PDF)

\section{AUTHOR INFORMATION}

\section{Corresponding Author}

*E-mail: pellegrino@iit.it. ORCID *

Bruce E. Cohen: 0000-0003-3655-3638

Teresa Pellegrino: 0000-0001-5518-1134

\section{Present Address}

\#Dr. Manuel Pernia Lealis, Departamento de Química Orgánica y Farmacéutica, Universidad de Sevilla, 41012 Seville (Spain).

\section{Author Contributions}

${ }^{\ddagger}$ Maria Elena Materia and Manuel Pernia Leal contributed equally.

\section{Notes}

The authors declare no competing financial interest.

\section{ACKNOWLEDGMENTS}

Work at the Molecular Foundry was supported by the Office of Science, Office of Basic Energy Sciences, of the U.S. Department of Energy under Contract No. DE-AC0205CH11231. The work at IIT was partially funded by COMPASS project, by AIRC project (Contract No. 14527), and by European Research Council (starting grant ICARO, Contract No. 678109). M.P.L. thanks to the V Plan Propio of the University of Seville for the Postdoctoral Fellowship. The authors thank D. Subramanian, E. Levy, and T. Bischof for assistance with UCNP synthesis and characterization.

\section{REFERENCES}

(1) Haase, M., and Schäfer, H. (2011) Upconverting Nanoparticles. Angew. Chem., Int. Ed. 50, 5808-5829.

(2) Wang, F., Banerjee, D., Liu, Y., Chen, X., and Liu, X. (2010) Upconversion nanoparticles in biological labeling, imaging, and therapy. Analyst 135, 1839-1854.

(3) Wu, S., Han, G., Milliron, D. J., Aloni, S., Altoe, V., Talapin, D. V., Cohen, B. E., and Schuck, P. J. (2009) Non-blinking and photostable upconverted luminescence from single lanthanide-doped nanocrystals. Proc. Natl. Acad. Sci. U. S. A. 106, 10917-10921.

(4) Wang, F., Han, Y., Lim, C. S., Lu, Y., Wang, J., Xu, J., Chen, H., Zhang, C., Hong, M., and Liu, X. (2010) Simultaneous phase and size control of upconversion nanocrystals through lanthanide doping. Nature 463, 1061-1065.

(5) Park, Y. I., Lee, K. T., Suh, Y. D., and Hyeon, T. (2015) Upconverting nanoparticles: a versatile platform for wide-field twophoton microscopy and multi-modal in vivo imaging. Chem. Soc. Rev. 44, 1302-17.

(6) Levy, E. S., Tajon, C. A., Bischof, T. S., Iafrati, J., FernandezBravo, A., Garfield, D. J., Chamanzar, M., Maharbiz, M. M., Sohal, V. S., Schuck, P. J., et al. (2016) Energy-Looping Nanoparticles: Harnessing Excited-State Absorption for Deep-Tissue Imaging. ACS Nano 10, 8423-8433.

(7) Chan, E. M., Levy, E. S., and Cohen, B. E. (2015) Rationally Designed Energy Transfer in Upconverting Nanoparticles. Adv. Mater. 27 (38), 5753-5761. 
(8) Liu, Y., Tu, D., Zhu, H., and Chen, X. (2013) Lanthanide-doped luminescent nanoprobes: controlled synthesis, optical spectroscopy, and bioapplications. Chem. Soc. Rev. 42, 6924-6958.

(9) Zhang, H., Li, Y., Ivanov, I. A., Qu, Y., Huang, Y., and Duan, X. (2010) Plasmonic modulation of the upconversion fluorescence in $\mathrm{NaYF} 4: \mathrm{Yb} / \mathrm{Tm}$ hexaplate nanocrystals using gold nanoparticles or nanoshells. Angew. Chem., Int. Ed. 49, 2865-8.

(10) Cheng, L., Yang, K., Li, Y., Chen, J., Wang, C., Shao, M., Lee, S.T., and Liu, Z. (2011) Facile Preparation of Multifunctional Upconversion Nanoprobes for Multimodal Imaging and DualTargeted Photothermal Therapy. Angew. Chem., Int. Ed. 50, 73857390.

(11) Liu, C., Gao, Z., Zeng, J., Hou, Y., Fang, F., Li, Y., Qiao, R., Shen, L., Lei, H., Yang, W., and Gao, M. (2013) Magnetic/ Upconversion Fluorescent NaGdF4:Yb,Er Nanoparticle-Based DualModal Molecular Probes for Imaging Tiny Tumors in Vivo. ACS Nano 7, 7227-7240.

(12) Johnson, N. J. J., Oakden, W., Stanisz, G. J., Scott Prosser, R., and van Veggel, F. C. J. M. (2011) Size-Tunable, Ultrasmall NaGdF4 Nanoparticles: Insights into Their T1MRI Contrast Enhancement. Chem. Mater. 23, 3714-3722.

(13) Cheng, L., Wang, C., Ma, X., Wang, Q., Cheng, Y., Wang, H., Li, Y., and Liu, Z. (2013) Multifunctional Upconversion Nanoparticles for Dual-Modal Imaging-Guided Stem Cell Therapy under Remote Magnetic Control. Adv. Funct. Mater. 23, 272-280.

(14) Challenor, M., Gong, P., Lorenser, D., House, M. J., Woodward, R. C., St, St. Pierre, T., Fitzgerald, M., Dunlop, S. A., Sampson, D. D., and Iyer, K. S. (2014) The influence of NaYF4:Yb,Er size/phase on the multimodality of co-encapsulated magnetic photon-upconverting polymeric nanoparticles. Dalton transactions (Cambridge, England: 2003) 43, 16780-7.

(15) Li, X., Zhao, D., and Zhang, F. (2013) Multifunctional Upconversion-Magnetic Hybrid Nanostructured Materials: Synthesis and Bioapplications. Theranostics 3, 292-305.

(16) Xu, H., Cheng, L., Wang, C., Ma, X. X., Li, Y. G., and Liu, Z. (2011) Polymer encapsulated upconversion nanoparticle/iron oxide nanocomposites for multimodal imaging and magnetic targeted drug delivery. Biomaterials 32, 9364-9373.

(17) Di Corato, R., Bigall, N. C., Ragusa, A., Dorfs, D., Genovese, A., Marotta, R., Manna, L., and Pellegrino, T. (2011) Multifunctional Nanobeads Based on Quantum Dots and Magnetic Nanoparticles: Synthesis and Cancer Cell Targeting and Sorting. ACS Nano 5, 11091121.

(18) Ostrowski, A. D., Chan, E. M., Gargas, D. J., Katz, E. M., Han, G., Schuck, P. J., Milliron, D. J., and Cohen, B. E. (2012) Controlled synthesis and single-particle imaging of bright, sub-10 nm lanthanidedoped upconverting nanocrystals. ACS Nano 6, 2686-2692.

(19) Pernia Leal, M., Torti, A., Riedinger, A., La Fleur, R., Petti, D. Cingolani, R., Bertacco, R, and Pellegrino, T. (2012) Controlled release of doxorubicin loaded within magnetic thermo-responsive nanocarriers under magnetic and thermal actuation in a microfluidic channel. ACS Nano 6, 10535-10545.

(20) Wang, F., and Liu, X. (2009) Recent advances in the chemistry of lanthanide-doped upconversion nanocrystals. Chem. Soc. Rev. 38, 976-989.

(21) Gu, Z., Yan, L., Tian, G., Li, S., Chai, Z., and Zhao, Y. (2013) Recent Advances in Design and Fabrication of Upconversion Nanoparticles and Their Safe Theranostic Applications. Adv. Mater. $25,3758-3779$.

(22) Deka, S. R., Quarta, A., Di Corato, R., Falqui, A., Manna, L., Cingolani, R., and Pellegrino, T. (2010) Acidic pH-Responsive Nanogels as Smart Cargo Systems for the Simultaneous Loading and Release of Short Oligonucleotides and Magnetic Nanoparticles. Langmuir 26, 10315-10324.

(23) Hilger, I., and Kaiser, W. A. (2012) Iron oxide-based nanostructures for MRI and magnetic hyperthermia. Nanomedicine 7, $1443-1459$.

(24) Lee, N., Kim, H., Choi, S. H., Park, M., Kim, D., Kim, H.-C., Choi, Y., Lin, S., Kim, B. H., Jung, H. S., et al. (2011) Magnetosome- like ferrimagnetic iron oxide nanocubes for highly sensitive MRI of single cells and transplanted pancreatic islets. Proc. Natl. Acad. Sci. U. S. A. 108, 2662-2667.

(25) Lee, N., Choi, Y., Lee, Y., Park, M., Moon, W. K., Choi, S. H., and Hyeon, T. (2012) Water-Dispersible Ferrimagnetic Iron Oxide Nanocubes with Extremely High r2 Relaxivity for Highly Sensitive in Vivo MRI of Tumors. Nano Lett. 12, 3127-3131.

(26) Pöselt, E., Kloust, H., Tromsdorf, U., Janschel, M., Hahn, C., Maßlo, C., and Weller, H. (2012) Relaxivity Optimization of a PEGylated Iron-Oxide-Based Negative Magnetic Resonance Contrast Agent for T2-Weighted Spin-Echo Imaging. ACS Nano 6, 16191624.

(27) Di Corato, R., Palumberi, D., Marotta, R., Scotto, M., CarregalRomero, S., Rivera Gil, P., Parak, W. J., and Pellegrino, T. (2012) Magnetic Nanobeads Decorated with Silver Nanoparticles as Cytotoxic Agents and Photothermal Probes. Small 8, 2731-2742.

(28) Yu, W. W., Falkner, J. C., Yavuz, C. T., and Colvin, V. L. (2004) Synthesis of monodisperse iron oxide nanocrystals by thermal decomposition of iron carboxylate salts. Chem. Commun., 2306-2307.

(29) Zeng, H., Rice, P. M., Wang, S. X., and Sun, S. H. (2004) Shapecontrolled synthesis and shape-induced texture of MnFe2O4 nanoparticles. J. Am. Chem. Soc. 126, 11458-11459.

(30) Carbone, L., Nobile, C., De Giorgi, M., Sala, F. D., Morello, G., Pompa, P., Hytch, M., Snoeck, E., Fiore, A., Franchini, I. R., et al. (2007) Synthesis and Micrometer-Scale Assembly of Colloidal CdSe/ CdS Nanorods Prepared by a Seeded Growth Approach. Nano Lett. 7, 2942-2950.

(31) Dabbousi, B. O., RodriguezViejo, J., Mikulec, F. V., Heine, J. R., Mattoussi, H., Ober, R., Jensen, K. F., and Bawendi, M. G. (1997) $\mathrm{CdSe}) \mathrm{ZnS}$ core-shell quantum dots: Synthesis and characterization of a size series of highly luminescent nanocrystallites. J. Phys. Chem. B 101, 9463-9475.

(32) Kalles, V., Zografos, G., Provatopoulou, X., Koulocheri, D., and Gounaris, A. (2013) The current status of positron emission mammography in breast cancer diagnosis. Breast Cancer 20, 123-130.

(33) Barrett, B. J., Parfrey, P. S., McDonald, J. R., Hefferton, D. M., Reddy, E. R., and McManamon, P. J. (1992) Nonionic low-osmolality versus ionic high-osmolality contrast material for intravenous use in patients perceived to be at high risk: Randomized trial. Radiology 183, $105-110$. 